\title{
Solving the inverse scattering problem with differential evolution: an experimental validation
}

\author{
Lucas H. Negri ${ }^{1}$, Aleksander S. Paterno ${ }^{2}$, Marcelo A. Pedroso ${ }^{3}$, Marcia Muller ${ }^{3}$ and José Luís Fabris ${ }^{3}$ \\ ${ }^{1}$ Federal Institute of Education, Science and Technology of Mato Grosso do Sul, Aquidauana, MS, Brazil \\ e-mail: lucas.negri@ifms.edu.br \\ ${ }^{2}$ Santa Catarina State University, Joinville, SC, Brazil email: aleksander.paterno@udesc.br \\ ${ }^{3}$ Federal University of Technology-Paraná, Curitiba, PR, Brazil emails: engmarcelol@gmail.com, \\ mmuller@utfpr.edu.br,fabris@utfpr.edu.br
}

\begin{abstract}
This work shows the experimental validation of a method developed for determining the strain profile applied to a fiber Bragg grating by solving the inverse scattering problem. The non-uniform strain profile is recovered by formulating an optimization problem, solved with an algorithm based on differential evolution. The method has proved to be able of recovering the shape and approximate amplitude of the actual strain profile applied to the FBG, opening new perspectives for optical sensing with fiber Bragg gratings.
\end{abstract}

Index Terms - Computational Intelligence, Differential Evolution, Fiber Bragg Gratings, Optical Sensors, Strain Sensing.

\section{INTRODUCTION}

Nowadays, fiber Bragg gratings (FBGs) are widely used in strain and temperature sensing systems [1]. The FBG consists of a periodic refractive index modulation, usually produced in the core of an optical fiber by the incidence of a UV-laser interference pattern. FBGs operate as selective mirrors, back-reflecting those wavelengths of the light propagating in the fiber core that satisfy the Bragg condition. Mechanical deformations and changes in temperature affect the FBG periodicity and the refractive index modulation, changing the Bragg condition and, consequently, the FBG reflection spectrum. Spectral shifts of the FBG resonance are the basis for the device operation as sensor. Most techniques of interrogation are based on algorithms used to detect the peak position of the FBG resonance [2],[3]. This strategy allows, e.g., punctual or quasi-distributed sensing of strain and temperature [1], [4]-[6] if the spectral wavelength shifts result from a uniform FBG deformation or a temperature change that affects the whole grating. Nevertheless, a non-uniform profile of strain or temperature results in additional spectral alterations such as broadening, splitting and asymmetrical shifts of the FBG resonance [7]. Such spectral features are usually detrimental for the sensor operation in most of the cases, but may become an asset if properly addressed. A non-uniform profile of strain or temperature applied to the FBG can be recovered by solving the inverse scattering problem, allowing multi-point monitoring with a single FBG in a distributed sensing configuration. This approach can benefit quasi-distributed sensing systems, leading to a reduction in the number of sensors and to an improvement in the sensor resolution. 
Different methods have been proposed in the literature to solve the inverse scattering problem, which consists in determining, from the analysis of the reflection spectrum, alterations in the structural parameters of a perturbed FBG [8]. These methods deal with specific situations in which monotonic strain profiles [9], information about the phase of the FBG spectrum [8], [10], [11], or even multiple FBGs [12] are required. In another work, an optimization algorithm is proposed to recover FBG parameters, in which a known strain profile is required as input to enhance the design sensitivity [13].

In this paper, the main contribution is the experimental validation of a method based on differential evolution and regularization, previously implemented and evaluated using simulations [14]. For this purpose, the approach was applied for determining the non-uniform strain profiles applied to an FBG. The structural parameters were recovered providing the successfully experimental validation of the method.

\section{METHODOLOGY}

\section{A. Determination of the Strain Profile}

Alterations are expected in the reflection spectrum of an FBG subjected to a mechanical deformation along the fiber length. Spectral changes are related to modifications in both grating pitch $\Lambda$ and mean value of the core refractive index $\bar{n}$ due to the photoelastic effect [7]. For a uniform FBG, these parameters depend on the applied strain $\varepsilon$, according to (1) and (2):

$$
\begin{gathered}
\Lambda^{\prime}(l)=\Lambda(l)(1+\varepsilon), \\
\bar{n}^{\prime}=\bar{n}-0.5 \bar{n}^{3}\left[p_{12}-v\left(p_{11}+p_{12}\right)\right] \varepsilon,
\end{gathered}
$$

where $l$ is the fiber length, $p_{11}$ and $p_{12}$ are the fiber photoelastic coefficients and $v$ is the Poisson coefficient of the silica [7].

Differential evolution, used to solve the inverse scattering problem, allows determining the unknown strain profile applied to the FBG by using characteristics of the unstrained grating as well as possible apodization parameters. Among these characteristics are the amplitude and periodicity of the refractive index modulation and the grating length. The algorithm simulates the FBG reflection spectrum by means of the transfer-matrix method, seeking for a solution that best matches the spectrum experimentally obtained. Additionally, due to the ambiguity resulting from the lack of phase information, regularization methods are used to obtain the convergence for the simplest solution.

For the experimental validation of the method, the FBG subjected to an unknown strain profile is considered as composed of $(N=10)$ different sections uniformly strained. A population of 40 candidate solutions is evolved using the adaptive method JADE [15]. Each one of the candidate solutions contains 10 characteristic parameters of the strain applied to the $(N=10)$ sections. The candidate solution with the largest fitness value is then selected. The number of sections can be chosen according to the application to obtain the required resolution. Nevertheless, as this number increases, the dimensionality of the problem also increases impairing the convergence of the method. 
For a candidate solution, the fitness calculation begins by the application of the corresponding strain profile. The parameters of each FBG section are introduced into (1) and (2) for $\Lambda$ and $\bar{n}$, respectively. The range of strain was established from 0 to $2.5 \mathrm{~m} \varepsilon$ based on typical values found in literature [7], [12]. Then, the FBG reflection spectrum is calculated and compared with the one experimentally measured. The mean absolute error is used as metric in the comparison. After the random generation of the initial population, the evolutionary process took 300 iterations. As discussed in previous works [7], [12], if the information about the phase is missing, a number of different strain profiles can be recovered from the same reflection spectrum. Nevertheless, this drawback can be avoided by adding two terms of regularization. The first one subtracts the norm $L_{1}$ of the solution $\left(L_{1}\right.$, multiplied by a coefficient $C_{l 1}$ ) from the fitness, providing solutions preferentially close to 0 . The second one subtracts the total variation of the solution ( $T V$ multiplied by a coefficient $C_{T V}$ ) from the fitness. The norm $L_{1}$ and the total variation $T V$ of a candidate solution are obtained from the $q_{i}$ terms according to:

$$
\begin{gathered}
L_{1}(q)=\sum_{i=1}^{N}\left|q_{i}\right|, \\
T V(q)=\sum_{i=2}^{N}\left|q_{i}-q_{i-1}\right| .
\end{gathered}
$$

The regularization technique works by favoring smooth/simple strain profiles over rough/complicated ones. A similar approach is usually employed for the FBG design to avoid refractive index profiles that are hard to fabricate and/or optimize [16].

\section{B. Experimental Setup}

In order to produce a non-uniform longitudinal deformation along the FBG, only a portion of the grating corresponding to approximately half of its total length was strained. A diagram of the experimental setup is shown in Fig. 1.

The fiber section with the FBG was fixed with self-adhesive resin cement (3M, RelyX U200) to the moving $(A)$ and fixed $(B)$ parts of the experimental apparatus. About the first half of the grating was fastened to the linear translation stage in $A$. Then, the fiber containing the free portion of the FBG was attached to the fix platform in $B$ at a distance $L_{p}$ from $A$. With this setup, the fiber segment with length $L_{p}$ that contains the FBG free portion can be submitted to longitudinal elongations.

The FBG with Bragg wavelength $\lambda_{B}$ at $1534 \mathrm{~nm}$ was produced at the Photorefractive Devices Unit of the Federal University of Technology - PR in a standard single mode fiber by the direct illumination of a phase mask [17]. In the recording setup, the FBG with length $l$ of $8.00 \mathrm{~mm}$ was written by scanning the laser beam (KrF, Xantos XS-Coherent, $248 \mathrm{~nm})$ along the phase mask (10 $\mathrm{mm}$ long, pitch of $1060 \mathrm{~nm}$ ).

The fiber segment with initial length $L_{p}$ of $106.1 \mathrm{~mm}$, localized between points $A$ and $B$ indicated in Fig. 1, was successively stretched in steps of $0.05 \mathrm{~mm}$ up to a total elongation $\Delta_{L}$ of $0.20 \mathrm{~mm}$. The system resolution was limited to $0.01 \mathrm{~mm}$ by the electronically controlled micrometer driver. 


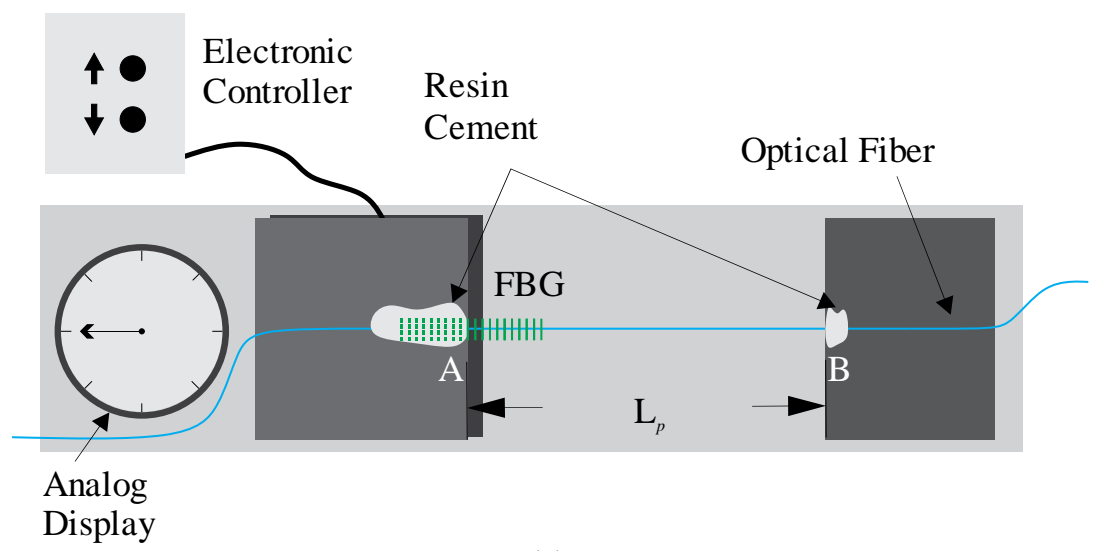

(a)

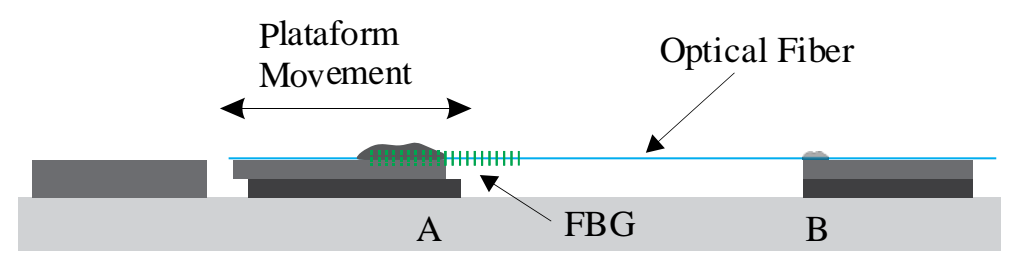

(b)

Fig. 1. Schematic diagram of the experimental setup:(a) top view and (b) side view. Elements in the diagram are out of scale.

FBG spectrum was recorded after each step by an optical spectrum analyzer (Anritsu MS9710B, resolution of $0.1 \mathrm{~nm}, 1001$ samples, 50 acquisitions per sample). The FBG reflection spectrum for the initial fiber length $L_{p}$ was taken as reference. Experiments were carried out at a controlled temperature of $(22.0 \pm 0.5){ }^{\circ} \mathrm{C}$ to avoid the influence of temperature changes in the FBG response due to the grating cross-sensitivity to both parameters, temperature and longitudinal strain.

As resin cements shrinks during its polymerization, half of the FBG is already strained at the initial conditions of the experiment. Therefore, the reference spectrum carries information about a permanent deformation, which is further considered for the determination of the strain profiles.

Once the experimental setup was ready for use, the method was first applied to determine the FBG initial parameters shown in Table I by using the reference spectrum.

TABLE I. FBG INITIAL PARAMETERS
\begin{tabular}{cc}
\hline Parameter & Value \\
\hline$l$ & $8.00 \mathrm{~mm}$ \\
$\Lambda$ & $526.474 \mathrm{~nm}$ \\
$\Delta_{n}$ & $41.505 \times 10^{-6}$ \\
\hline
\end{tabular}

After being submitted to consecutive elongations, the recorded FBG spectral data were used to reconstruct the applied strain profiles. The coefficients $C_{l l}=0.1$ and $C_{T V}=5.0$ of the regularization terms were experimentally determined by using a distinct data set. 


\section{RESULTS}

Fig. 2 shows a comparison between the FBG experimental (blue dashed lines) and simulated (orange continuous lines) reflection spectra obtained for the grating under the initial conditions and submitted to elongations $\Delta_{L}$ of $0.05,0.10,0.15$ and $0.20 \mathrm{~mm}$. As previously discussed, resin cement shrinks during the polymerization resulting in the optical fiber contraction. Consequently, Bragg resonance splits in two peaks that are present even in the FBG reference spectrum (unstrained fiber), corresponding to the Bragg wavelengths of FBG bonded and free portions.

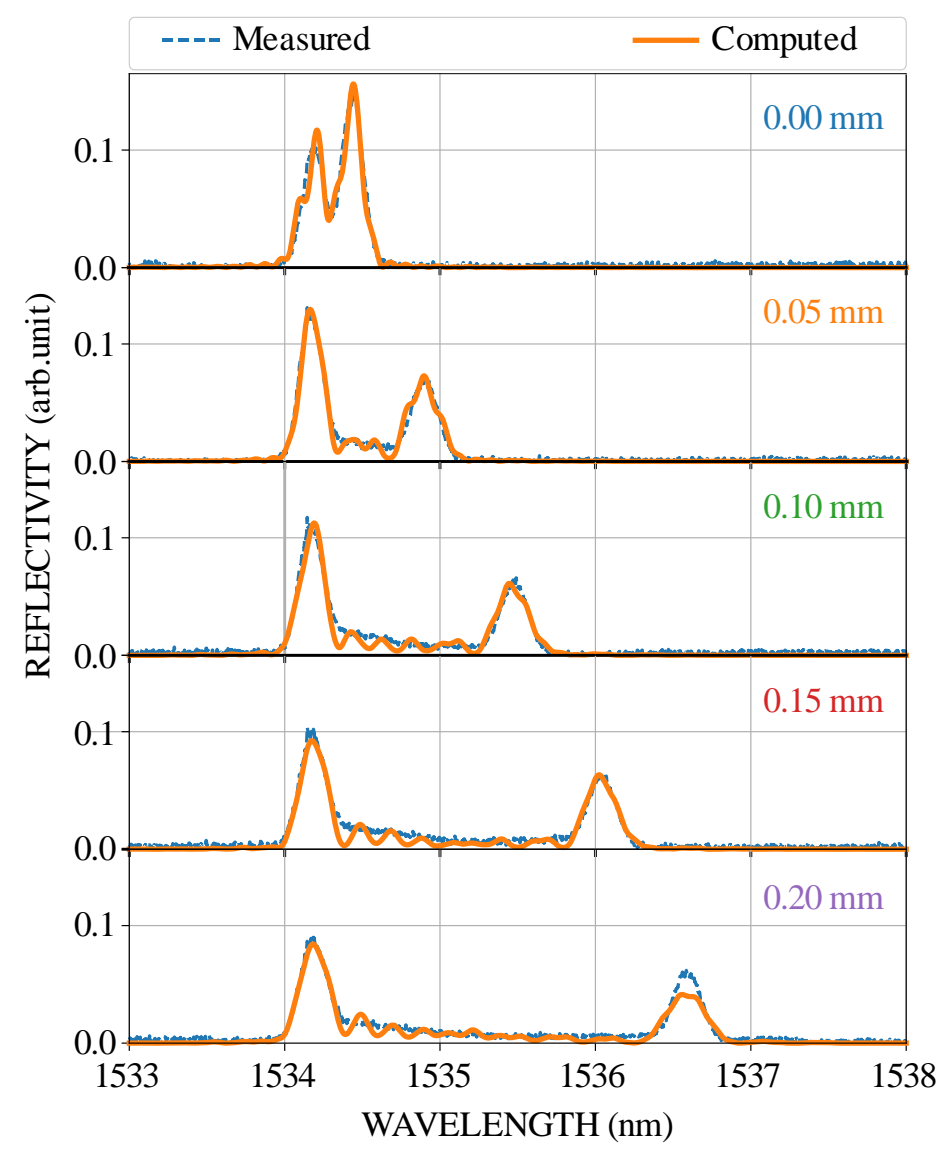

Fig. 2. Experimental (blue dashed line) and simulated (orange continuous line) reflection spectra obtained for the FBG under different strain conditions.

The parameters shown in Table I correspond to the FBG at the initial conditions and were calculated assuming $p_{11}=0.113, p_{12}=0.252, v=0.160$ and $\bar{n}=1.457$. As the fiber elongation takes place, the resonance of the free portion is red-shifted while the resonance related to the bonded portion remains at the same spectral position. Besides, spectra exhibit a broad band of reflection with lower intensity superposed to the two main resonances, an indicative of a non-uniform strain profile. On the basis of the used experimental setup, an approximate step-strain profile is expected as about half of the FBG was bonded and cannot be further deformed.

As can be seen from the graphs, the FBG spectra are efficiently estimated by the method based on differential evolution. Small divergences, which do not impair the result, are present only in the spectral range between the two main peaks. These differences result from the OSA resolution and the 
chosen reduced number of FBG regions considered for the simulations. Curves of Fig. 3 depict the reconstructed strain profiles applied to the FBG when the fiber is lengthened. Each one of the 10 FBG sections is subjected to a different strain determined by the method.

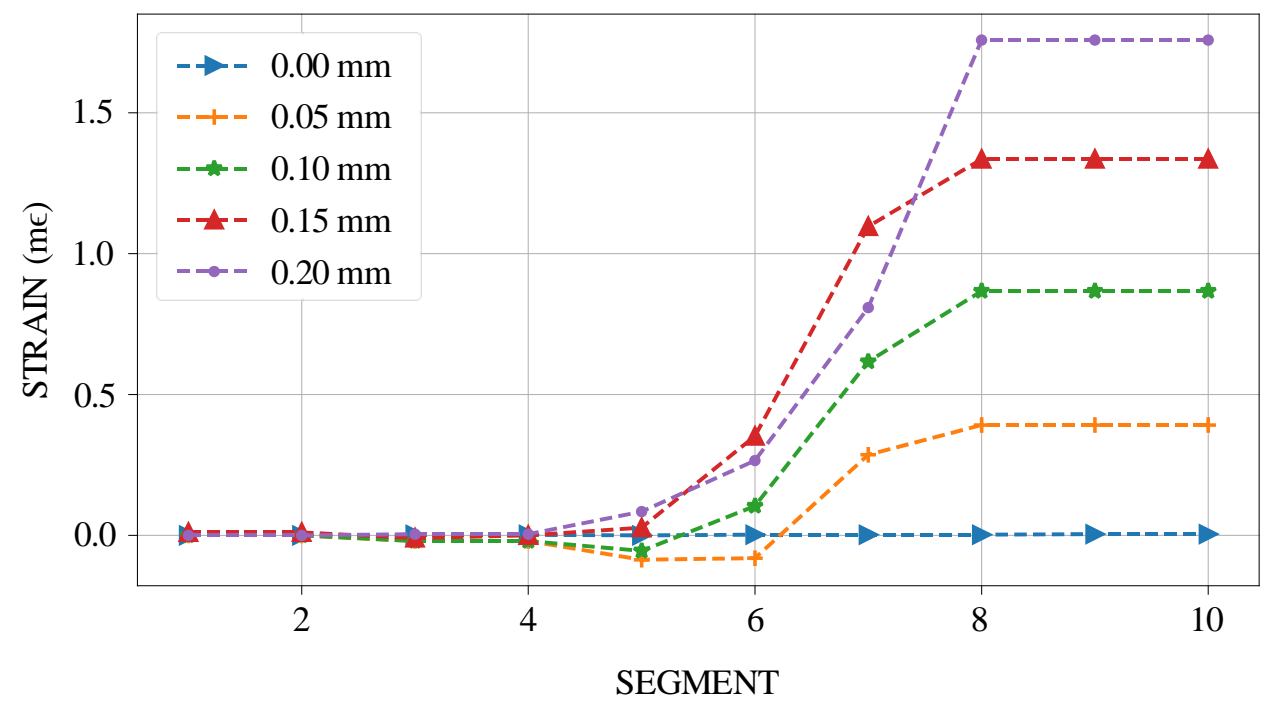

Fig. 3. FBG strain profiles reconstructed by the proposed method.

Table II presents the determined strain values as well as the expected strain obtained by the relation between the fiber elongation $\Delta_{L}$ and the initial length $L_{p}$. As previously discussed, the strain values in Table II were determined by considering the reference spectrum of the FBG obtained with half of the grating pre-strained due to the resin cement contraction.

TABLE II. STRAIN DETERMINED BY THE METHOD FOR EACH ONE OF THE 10 FBG SECTIONS AND THE EXPECTED STRAIN OBTAINED BY THE RATIO BETWEEN THE ELONGATION $\Delta_{L}$ AND THE INITIAL LENGTH $L_{P}$.

\begin{tabular}{|c|c|c|c|c|c|c|c|c|c|c|c|c|}
\hline \multirow[b]{2}{*}{$\Delta_{L}(\mathrm{~mm})$} & \multirow[b]{2}{*}{ Fiber section } & \multicolumn{10}{|c|}{$\begin{array}{c}\text { Computed Strain } \\
(\mathrm{m} \varepsilon)\end{array}$} & \multirow[t]{2}{*}{$\begin{array}{c}\text { Expected Strain } \\
(\mathrm{m} \varepsilon)\end{array}$} \\
\hline & & 1 & 2 & 3 & 4 & 5 & 6 & 7 & 8 & 9 & 10 & \\
\hline & 0.00 & 0.00 & 0.00 & 0.00 & 0.00 & 0.00 & 0.00 & 0.00 & 0.00 & 0.00 & 0.00 & 0.00 \\
\hline & 0.05 & 0.00 & 0.00 & -0.02 & -0.02 & -0.09 & -0.08 & 0.29 & 0.39 & 0.39 & 0.39 & 0.47 \\
\hline & 0.10 & 0.00 & 0.00 & -0.02 & -0.02 & -0.05 & 0.10 & 0.62 & 0.87 & 0.87 & 0.87 & 0.94 \\
\hline & 0.15 & 0.01 & 0.01 & -0.01 & 0.00 & 0.03 & 0.35 & 1.10 & 1.34 & 1.34 & 1.34 & 1.41 \\
\hline & 0.20 & 0.00 & 0.00 & 0.00 & 0.00 & 0.08 & 0.27 & 0.81 & 1.76 & 1.76 & 1.76 & 1.89 \\
\hline
\end{tabular}

By comparing the expected values of strain with the corresponding maximum values recovered by the method, relative changes from approximately $5 \%$ for an elongation of $0.15 \mathrm{~mm}$ up to $17 \%$ for an elongation of $0.05 \mathrm{~mm}$ were obtained.

As shown in Fig. 4, the reconstructed strain profile has a smooth transition between the free and attached regions. This result is expected both by the existence of a soft transition in the bonded region (as depicted in Fig. 4), that would prevent an abrupt change in the strain profile, as well as by the usage of regularization terms in the optimization algorithm. 


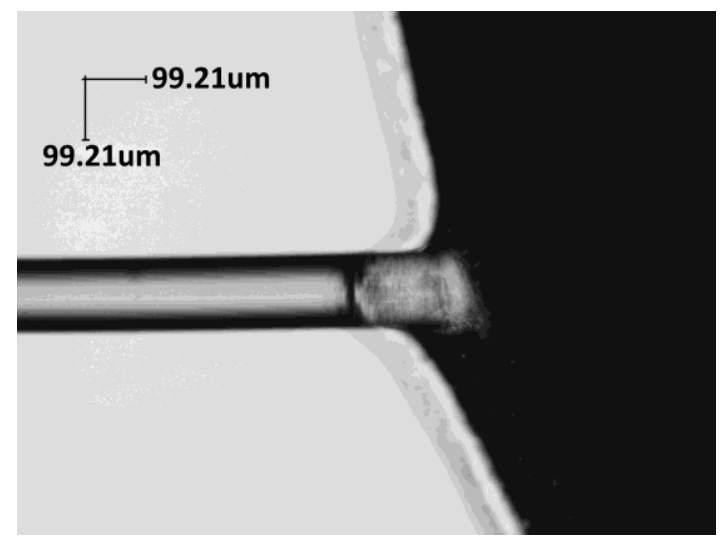

Fig. 4. Microscope image (top view) showing a soft transition between the FBG free and bonded sections.

\section{CONCLUSION}

The method based on differential evolution and regularization proposed in [14] was experimentally validated, providing an efficient reconstruction of strain profiles applied to an FBG. By recovering both the shape and amplitude of the strain profile using only the magnitude of the FBG reflection spectrum as input, the method allows FBGs application in distributed sensing.

\section{ACKNOWLEDGMENT}

The authors would like to thank the financial support obtained from the Brazilian Agencies: CAPES, CNPq, FINEP and Fundação Araucária.

\section{REFERENCES}

[1] A. D. Kersey, M. Davis, H. Patrick, M. LeBlanc, K. Koo, C. Askins, M. Putnam, and E. Friebele, "Fiber grating sensors," Journal of Lightwave Technology, vol. 15, no. 8, pp. 1442-1463, 1997.

[2] L. Negri, A. Nied, H. Kalinowski, and A. Paterno, "Benchmark for Peak Detection Algorithms in Fiber Bragg Grating Interrogation and a New Neural Network for its Performance Improvement," Sensors, vol. 11, no. 12, pp. 3466-3482, 2011.

[3] D. Tosi, M. Olivero, and G. Perrone, "Performance analysis of peak tracking techniques for fiber Bragg grating interrogation systems," Journal of Microwaves, Optoelectronics and Electromagnetic Applications, vol. 11, pp. 252$262,2012$.

[4] Y.-J. Rao, "In-fibre Bragg grating sensors," Meas. Sci. Technol., vol. 8, n. 4, pp. 355-375, 1997.

[5] N. Hirayama, Y. Sano, "Fiber Bragg grating temperature sensor for practical use," ISA Trans., vol. 39, no. 2, pp. 169$173,2000$.

[6] J.-S. Heo, J.-H. Chung, and J.-J. Lee, "Tactile sensor arrays using fiber Bragg grating sensors," Sensors Actuators A Phys., vol. 126, no. 2, pp. 312-327, 2006.

[7] S. Huang, M. Leblanc, M. M. Ohn, and R. M. Measures, "Bragg intragrating structural sensing," Applied Optics, vol. 34, no. 22, pp. 5003-5009, 1995.

[8] J. Skaar and R. Feced, "Reconstruction of gratings from noisy reflection data," Journal of the Optical Society of America. A: Optics and Image Science, and Vision, vol. 19, no. 11, pp. 2229-2237, 2002.

[9] M. Leblanc, S. Y. Huang, M. Ohn, R. M. Measures, A. Guemes, and A. Othonos, "Distributed strain measurement based on a fiber Bragg grating and its reflection spectrum analysis," Optics Letters, vol. 21, no. 17, pp. 1405-1407, 1996.

[10] M. Ohn, S. Huang, R. Measures, and J. Chwang, "Arbitrary strain profile measurement within fibre gratings using interferometric Fourier transform technique,” Electronics Letters, vol. 33, no. 14, pp. 1242-1243, 1997.

[11] M. A. Muriel, J. Azaña, and A. Carballar, "Fiber grating synthesis by use of time-frequency representations," Optics Letters, vol. 23, no. 19, pp. 1526-1528, 1998.

[12] H.-C. Cheng and Y.-L. Lo, "Arbitrary strain distribution measurement using a genetic algorithm approach and two fiber Bragg grating intensity spectra," Optics Communications, vol. 239, no. 4-6, pp. 323-332, 2004.

[13] C. C. Cheng, Y. L. Lo, W. Y. Li, C. T. Kuo, and H. C. Cheng, "Estimations of fiber Bragg grating parameters and strain gauge factor using optical spectrum and strain distribution information," Appl. Opt., vol. 46, no. 21, pp. 4555-4562, 2007. 
[14] L. H. Negri, H. S. Lopes, M. Muller, J. L. Fabris, and A. S. Paterno, "An efficient method to determine strain profiles on FBGs by using differential evolution and GPU," in 2015 Latin America Congress on Computational Intelligence (LA-CCI), pp. 1-6, 2015.

[15] J. Zhang and A. C. Sanderson, "JADE: Adaptive Differential Evolution with Optional External Archive," IEEE Trans. Evol. Comput., vol. 13, no. 5, pp. 945-958, 2009.

[16] M. J. D. Sousa, J. C. W. A. Costa, R. M. D. Souza, and R. V. M. P. Pantoja, "FBG optimization using spline encoded evolution strategy," Journal of Microwaves, Optoelectronics and Electromagnetic Applications, vol. 10, pp. 165 - 178 , 2011.

[17] K. O. Hill, B. Malo, F. Bilodeau, D. C. Johnson, and J. Albert, "Bragg gratings fabricated in monomode photosensitive optical fiber by UV exposure through a phase mask,” Appl. Phys. Lett., vol. 62, no. 10, pp. 1035-1037, 1993. 